\title{
Caminhabilidade e envelhecimento saudável: uma proposta de análise para cidades brasileiras de pequeno e médio porte
}

\author{
Walkability and healthy aging: an analytical \\ proposal for small and medium-sized \\ Brazilian cities
}

\author{
Aline Siqueira Fogal Vegi 1 \\ Elpídio Inácio Fernandes Filho 1 \\ Milene Cristine Pessoa 2 \\ Karla Lisboa Ramos 3 \\ Andréia Queiroz Ribeiro 1
}

doi: 10.1590/0102-311X00215218

\section{Resumo}

Há evidências de que ambientes urbanos que desestimulam a caminhada contribuem para a incapacidade funcional de idosos. Vários indices foram propostos para descrever a caminhabilidade de uma área combinando aspectos do ambiente construído que promovem ou inibem a caminhada. No entanto, devido a problemas de qualidade e disponibilidade de dados no Brasil, até o momento não há um índice de caminhabilidade aplicável a todas as cidades do país e devidamente testado na população. O objetivo deste estudo foi propor um indice de caminhabilidade baseado em sistemas de informação geográfica para uma cidade de médio porte, com dados de livre acesso, bem como testar sua associação com a incapacidade funcional em idosos. Foram usados os dados da área urbana de um município de médio porte para selecionar um conjunto parcimonioso de variáveis por meio de análise fatorial. O índice obtido foi testado em relação à sua associação com a capacidade para a realização de atividades de vida diária que requerem maior movimentação, em 499 idosos utilizando equações de estimativas generalizadas. O indice de caminhabilidade resultante foi composto por densidade residencial, densidade comercial, conectividade de ruas, presença de calçadas e iluminação pública. Essas variáveis compuseram o primeiro fator da análise fatorial, excluindo-se apenas a arborização que ficou retida no segundo fator. Verificou-se que o pior escore de caminhabilidade estava associado ao maior escore de incapacidade funcional. Com base nos resultados e na validação deles, o estudo sugere um índice de caminhabilidade facilmente aplicável com grande potencial de uso em planos de ação para adequar os ambientes.

Idoso; Envelhecimento Saudável; Limitação da Mobilidade; Meio Ambiente e Saúde Pública; Saúde do Idoso

\author{
Correspondência \\ A. Q. Ribeiro \\ Universidade Federal de Viçosa. \\ Av. P. H. Rolfs s/n, Ed. Centro de Ciências Biológicas II, Viçosa, \\ MG 36570-900, Brasil. \\ andreia.ribeiro@ufv.br \\ 1 Universidade Federal de Viçosa, Viçosa, Brasil. \\ 2 Universidade Federal de Minas Gerais, Belo Horizonte, Brasil. \\ 3 Universidade de Brasília, Brasília, Brasil.
}




\section{Introdução}

A população idosa do mundo aumentou acentuadamente durante as últimas décadas, especialmente nos países da América Latina e do Caribe, como o Brasil 1. Nesse sentido, o envelhecimento populacional representa uma conquista, mas também uma responsabilidade para os gestores públicos e a sociedade. É crucial desenvolver estratégias que promovam a vida ativa nessa fase da vida com independência, autonomia e qualidade 2,3 .

Uma questão central nesse processo diz respeito à necessidade de se planejar comunidades urbanas adaptadas para os idosos. Para abordar esse tema, a Organização Mundial da Saúde (OMS) lançou o modelo Cidade Amiga do Idoso, que direciona a concepção de cidades que promovem "o envelhecimento ativo ao otimizar oportunidades para saúde, participação e segurança, para aumentar a qualidade de vida à medida que as pessoas envelhecem" 4 (p. 7). Tal modelo tem como conceito base de envelhecimento saudável o processo de promoção e manutenção da capacidade funcional, a partir do incentivo à participação econômica e social em um ambiente seguro e acessível 4 . Posteriormente, o documento intitulado Acción Multisectorial para un Envejecimiento Saludable Basado en el Ciclo de Vida: Proyecto de Estrategia y Plan de Acción Mundiales sobre el Envejecimiento y la salud 5, formulado e discutido durante a 69a Assembleia Mundial da Saúde em abril de 2016, estabeleceu as características e adaptações necessárias na infraestrutura e nos serviços das cidades para que os habitantes com mais de 60 anos tivessem suas necessidades atendidas e seus direitos assegurados. A ação então veio renovar o compromisso de concentrar a atenção nas necessidades e direitos dos idosos, traçando metas e estratégias para tornar as cidades inclusivas e acessíveis, especialmente para eles.

Particularmente, os idosos são mais susceptíveis ao seu ambiente próximo e são mais vulneráveis a mudanças cognitivas e físicas, o que pode diminuir sua capacidade de lidar com as características desfavoráveis do ambiente, podendo resultar na diminuição da sua capacidade de caminhar e, consequentemente, da capacidade funcional 3 . No entanto, pequenas modificações no ambiente físico podem ser úteis para manter a independência das pessoas idosas. Assim, ambientes adequados para os idosos ajudam a promover o envelhecimento ativo mantendo a capacidade intrínseca ao longo da vida e aumentando a capacidade funcional, de modo que as pessoas com diferentes graus de dificuldade possam ser independentes e autônomas 2,6.

Medidas destinadas a criar ambientes adequados para as pessoas mais velhas podem ser focadas em diferentes contextos. Uma das formas pela qual a vizinhança promove a manutenção e melhoria da capacidade funcional é atuando sobre a caminhada ao ar livre para diferentes propósitos - incluindo transporte, recreação e exercício 2,3. Nesse contexto, a caminhabilidade (do inglês walkability) é uma característica importante do ambiente urbano, especialmente em programas visando à manutenção da vida ativa e saudável dos idosos 2,7. Tal característica mede o quão convidativa é uma área para que o pedestre acesse diferentes partes da cidade a pé ou de bicicleta 8 . Assim, a caminhabilidade deve motivar mais pessoas a adotarem o caminhar como forma de deslocamento efetiva, restabelecendo suas relações interdependentes com as ruas e sua vizinhança. Há fortes evidências de que a caminhabilidade da vizinhança está relacionada à atividade física individual e ao transporte ativo não apenas entre os jovens e adultos 9,10,11, mas também em idosos 7,12,13,14,15. No entanto, quase todas essas evidências vêm de países de alta renda e foram pouco estudadas em idosos e com resultados inconsistentes na América Latina e no Brasil 16.

Nos últimos anos foram construídos e testados vários índices que avaliam a caminhabilidade 8,17, $18,19,20,21,22,23,24,25,26$. Essa variedade de índices tem sido justificada pelo fato de que tanto os atributos e sua quantificação para a criação do índice de caminhabilidade devem ser pensados especificamente para a população alvo 18 . Esses índices são compostos por diferentes variáveis do ambiente construído que podem promover ou inibir a caminhada em várias escalas espaciais, bem como em diferentes ambientes e para diferentes populações. No entanto, não há conhecimento, no Brasil, de um índice que pode ser aplicado em todo o território nacional com dados de livre acesso e baixa dificuldade operacional. Por isso, o objetivo deste trabalho foi propor um índice de caminhabilidade baseado em sistemas de informação geográfica (SIG) para uma cidade de médio porte, com dados de livre acesso para os municípios brasileiros, bem como testar sua associação com a incapacidade funcional em idosos. 


\section{Metodologia}

\section{Área de estudo}

A cidade de Viçosa, localizada na Zona da Mata do Estado de Minas Gerais, Brasil, teve, em 2009, uma população de aproximadamente 71.885 habitantes distribuídos em uma área de $299,397 \mathrm{~km}^{2}$ dividida em 99 setores censitários na região urbana e 11 na zona rural 27. O setor censitário é definido como uma área contínua, situada em um único quadro urbano ou rural, com dimensão e número de domicílios que permitam o levantamento das informações por um único agente do censo 27 . A área de abrangência do estudo foram os setores censitários urbanos da cidade de Viçosa, que apresentavam continuidade espacial, ou seja, estavam justapostos. Nas regiões urbanas, cada setor agrupa, em média, de 250 a 350 domicílios.

Para auxiliar na discussão, os setores censitários foram agrupados em 11 regiões de planejamento segundo adaptação do Retrato Social de Viçosa III 28. Tal divisão permite uma compreensão mais precisa da realidade local com base na agregação de locais com características mais homogêneas.

\section{Seleção de variáveis}

Baseando-se na revisão compreensiva da literatura, foi identificada e selecionada uma lista de variáveis baseadas em SIG que se mostrava relevante para a composição do índice de caminhabilidade. A busca foi realizada nas bases de dados TRIS, MEDLINE e Web of Science, usando-se índices de pedestres, caminhabilidade e ambiente construído como palavras-chave. Somente a literatura que mediu ou quantificou os correlatos do ambiente físico construído usando medidas objetivas e que apresentaram como desfecho a caminhada para o lazer ou transporte ou capacidade funcional de indivíduos adultos vivendo em bairros urbanos foi selecionada para revisão adicional. Nenhuma restrição de data de publicação foi imposta. As listas de referência de todas as publicações incluídas, assim como as listas de referência de revisões recentemente publicadas relacionadas ao ambiente construído e à caminhada também foram escaneadas. Foram extraídas as evidências que ligavam os atributos do ambiente relacionados à caminhabilidade. Posteriormente, as variáveis foram restritas àquelas para as quais as fontes de dados estavam prontamente disponíveis para a maioria dos municípios brasileiros, sem custo ou alta dificuldade operacional.

\section{Obtenção e criação de variáveis}

Com o objetivo de minimizar os erros de mensuração causados pela dispersão urbana, as densidades dos atributos de interesse foram calculadas de forma a desconsiderar os vazios urbanos por meio da utilização do comprimento das ruas e não pela área total de cada setor censitário como comumente é feito em outros estudos.

A malha de ruas do Município de Viçosa foi obtida no projeto Open Street Map (https://planet. openstreetmap.org/), que tem o mapeamento digital de todo o mundo, disponibilizado gratuitamente. Para a criação da variável comprimento de rua, foram excluídas estradas expressas, caminhos rurais e outros caminhos não adequados para a caminhada. Posteriormente, foi calculada a soma do comprimento das ruas em quilômetros dentro de cada setor censitário.

A conectividade das ruas foi obtida com base na contabilização de todas as conexões entre as ruas com número de nós reais (nós que estão em interseções de 4 vias ou 3 vias, desconsiderando ruas sem saída). Essa contagem foi dividida pelo comprimento de ruas de cada setor de forma a obter uma medida de densidade.

Utilizando os dados georreferenciados do Censo de 201027 foram calculadas a densidade residencial e a densidade de estabelecimentos comerciais. O número de residências de cada setor censitário e a contabilização de estabelecimentos não residenciais (excluindo-se garagens, depósitos, lotes vagos, lojas/cômodos vagos e construções e comércios agropecuários) foram divididos pelo total de quilômetros de ruas de cada um dos setores censitários avaliados. 
Por último, ainda usando a base de dados georreferenciada do Instituto Brasileiro de Geografia e Estatística (IBGE), utilizou-se os dados do entorno das residências relativos à presença de calçadas, iluminação pública e arborização. Essas informações foram incluídas pela primeira vez no Censo de 2010, na fase de pré-coleta, por meio de observações diretas realizadas pelos técnicos do IBGE 29. Os pesquisadores do Censo avaliaram se na face da quadra da residência existia calçada/passeio, ou seja, caminho calçado ou pavimentado, destinado à circulação de pedestres. De forma semelhante, pesquisaram se na face da quadra em trabalho ou na sua face confrontante existia pelo menos um ponto fixo (poste) de iluminação pública. A arborização foi verificada mediante a presença de árvore na face da quadra em trabalho, na sua face confrontante ou no canteiro central, ou seja, se havia árvore ao longo da calçada/passeio e/ou em canteiro que divide pistas de um mesmo logradouro, mesmo que apenas em parte. Considerou-se também a arborização quando existente em logradouros sem pavimentação e/ou sem calçada/passeio. Para as três características observadas, determinou-se o percentual de presença no setor dividindo-se o total de domicílios que tinham a característica pelo total de domicílios do setor e, então, multiplicando-se por 100. Como a coleta desses dados não foi baseada em uma contagem de atributos por setor censitário (comprimento, quantidade por residência), mas sim em uma variável dicotômica (presença ou ausência), não foi possível calcular tais características por área do setor ou comprimento das ruas.

\section{Criação do índice}

A análise fatorial exploratória (AFE) foi usada como a primeira etapa do desenvolvimento de um conjunto parcimonioso de medidas de caminhabilidade. Para tanto, foi utilizado o método de extração dos componentes principais e rotação varimax com a normalização de Kaiser. Para verificar o número de fatores a serem retidos, foram usados os critérios de Kaiser (eigenvalue > 1), Cattell (scree plot) e de Horn (análise paralela). Foram mantidos os itens cujas cargas fatoriais eram maiores ou iguais a 0,4 e escolhido o fator com a maior explicação da variância 30 .

A distribuição de cada variável ambiental que permaneceu no primeiro fator na análise fatorial foi transformada em escore z e somada de forma a compor o índice de caminhabilidade. Posteriormente, o índice foi dividido em quintis para permitir uma classificação da intensidade de caminhabilidade das áreas: muito baixa, baixa, média, alta ou muito alta.

\section{Validade preditiva}

A associação do índice proposto com a incapacidade funcional dos idosos foi testada usando-se os dados da pesquisa Condições de Saúde, Nutrição e Uso de Medicamentos por Idosos do Município de Viçosa (MG): Um Inquérito de Base Populacional, um estudo epidemiológico transversal de base populacional no Município de Viçosa, conduzido durante o ano de 2009 com 621 idosos não institucionalizados. Para o presente trabalho, foram avaliados os 499 idosos residentes em endereços localizados em setores censitários urbanos que apresentavam continuidade espacial, ou seja, estavam justapostos. Mais detalhes sobre a metodologia podem ser vistos em publicação anterior 31 .

Com base nas respostas fornecidas para o questionário de incapacidade funcional aplicado durante a pesquisa, foi criado um escore de incapacidade funcional baseado na teoria de resposta ao item (TRI) 30. Para isso, foram selecionadas nove atividades de vida diária que exigem um certo grau de movimentação para sua execução. Tais atividades foram: banhar-se, caminhar de um cômodo a outro dentro de casa e levantar-se da cama para uma cadeira, preparar os alimentos ou cozinhar, sair de casa ou tomar um ônibus, fazer compras, arrumar a casa, fazer trabalhos manuais domésticos e lavar e passar roupas. As opções de respostas para cada item eram: incapaz de fazê-lo; grande dificuldade; pequena dificuldade e sem dificuldade alguma. A consistência interna do escore com base nos itens mencionados anteriormente foi testada usando-se o alfa de Cronbach $(\alpha=0,96)$. O escore final do índice diz respeito à dificuldade de movimentação baseada na execução das atividades de vida diária avaliadas. Portanto, na medida em que o índice aumenta, maior a dificuldade apresentada pelos idosos.

O georreferenciamento do local da residência foi realizado após a entrevista dos idosos por meio do uso de um GPS (global positioning system) portátil. 
Para testar associações entre exposição (índice de caminhabilidade) e resultado (maior incapacidade funcional) foram utilizados modelos de equações de estimativa generalizadas (GEE). As análises foram realizadas usando-se a matriz de correlação de trabalho intercambiável. Assim, essa análise considera a estrutura de correlação entre as observações e, portanto, produz estimativas mais eficientes e imparciais para os parâmetros do modelo de regressão quando se trata de dados correlacionados, pois considera a possível dependência existente entre os participantes que residem no mesmo setor censitário. Além disso, o modelo GEE não requer a suposição de normalidade dos dados 32 . Nos modelos foram estimados os coeficientes $(\beta)$ bruto e ajustado por idade, sexo e renda, além dos respectivos intervalos de 95\% de confiança (IC95\%).

Nessa etapa, assim como para a AFE, os dados foram processados e analisados com o auxílio do programa SPSS para Windows, versão 24 (https://www.ibm.com/). O processamento de todos os dados geográficos e a confecção dos mapas foi realizado no software ArcGIS 10.3 (http://www.esri. com/software/arcgis/index.html).

\section{Resultados}

\section{Variáveis candidatas a compor o índice}

As variáveis relacionadas com a caminhada, identificadas por meio de revisão da literatura, estão resumidas no Quadro 1. Dessas, apenas as variáveis arborização, conectividade das ruas, densidade comercial, densidade residencial, iluminação pública e presença de calçadas têm dados de livre acesso para todos os municípios brasileiros.

Foi realizada a espacialização das variáveis candidatas ao índice permitindo a observação da distribuição das mesmas. Verificou-se que a iluminação pública foi a característica mais presente nos setores censitários, e a densidade comercial foi a mais heterogênea. As características presença de calçadas, densidade residencial, densidade comercial e arborização demandam modificações nessas áreas para torná-las mais atrativas. De forma geral, observou-se que a região central do município é a que apresenta a melhor infraestrutura para a caminhada e que as regiões mais periféricas contam com a pior distribuição dos componentes que facilitem esta prática (Figura 1).

\section{Seleção das variáveis - análise fatorial por componentes principais}

Inicialmente, verificou-se a fatoriabilidade da matriz de correlação entre os itens do índice de caminhabilidade por meio do índice Kaiser-Meyer-Olkin (KMO) e teste de esfericidade de Bartlett. O valor do KMO foi de 0,55 e o teste de Bartlett apresentou valor de $\mathrm{p}<0,001$, o que permite prosseguir com a análise fatorial. A AFE gerou dois fatores e indicou que o fator 1, com a maior explicação da variância total (35,89\%), agrupa cinco itens, sendo eles densidade residencial, densidade comercial, conectividade de ruas, presença de calçadas e de iluminação pública (Tabela 1).

\section{Índice de caminhabilidade}

Após a seleção das variáveis no item a, obteve-se o índice de caminhabilidade com base na equação:

ÍNDICE DE CAMINHABILIDADE $=[($ densidade residencial z-score $)+$

(\% de presença de calçadas $\mathrm{z}$-score $)+($ densidade comercial z-score $)+$

(\% de presença de iluminação pública z-score) + (densidade de intersecções z-score)]

Os valores do índice variaram entre -13,95 e 7,74, com mediana de 0,2431 e amplitude interquartil de 4,89. A Figura 2 apresenta a distribuição espacial da caminhabilidade na cidade de Viçosa em quintis. Verifica-se o predomínio de áreas de muito baixa e baixa caminhabilidade na cidade, com destaque para as regiões periféricas, tais como Silvestre, Amoras e Nova Viçosa. As áreas com os maiores índices estão concentradas na região do Centro e adjacências. 


\section{Quadro 1}

Atributos do ambiente e evidência científica relacionada à caminhabilidade.

\begin{tabular}{|c|c|c|}
\hline Atributos & Evidência científica & $\begin{array}{l}\text { Dados } \\
\text { disponíveis }\end{array}$ \\
\hline Arborização & $\begin{array}{l}\text { As árvores das ruas proporcionam configurações facilitadoras à caminhada como sombra nos dias } \\
\text { quentes e melhora da estética da vizinhança tornando a calçada mais atraente para os pedestres } 42,43\end{array}$ & Sim \\
\hline $\begin{array}{l}\text { Conectividade das } \\
\text { ruas }\end{array}$ & 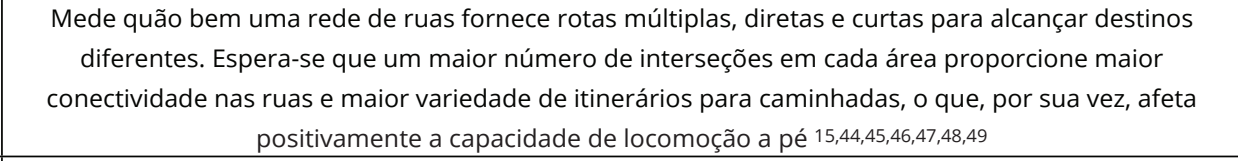 & Sim \\
\hline Crimes & $\begin{array}{l}\text { O número de ocorrências de delitos registrados indica o quanto os pedestres em circulação em } \\
\text { determinado local estão expostos a riscos relativos à segurança pública. A maior ocorrência está } \\
\text { associada com a menor chance de caminhada } 44,50,51,52\end{array}$ & Não \\
\hline Declividade & $\begin{array}{l}\text { A diferença de altura entre dois pontos ao longo de uma caminhada tem influência significativa na } \\
\text { energia necessária para andar entre eles estando a maior inclinação do terreno associada a menores } \\
\text { chances de caminhar } 44,53,54,55\end{array}$ & Não \\
\hline Densidade comercial & $\begin{array}{l}\text { Uma vez que sugere uma área onde muitos lugares estão a uma curta distância, um maior número de } \\
\qquad \text { lojas e empresas pode influenciar positivamente a mobilidade } 52,56\end{array}$ & Sim \\
\hline Densidade residencial & $\begin{array}{l}\text { A alta densidade residencial implica áreas mais comerciais e recreativas na proximidade de áreas de } \\
\text { habitação densas, influenciando positivamente a mobilidade } 47,49,55,56,57,58\end{array}$ & Sim \\
\hline Iluminação pública & $\begin{array}{l}\text { Calçadas e espaços públicos bem iluminados são essenciais para levar mais pessoas a aproveitarem } \\
\text { os espaços. Espaços ativos e bem iluminados não são propícios ao crime e aumentam a sensação de } \\
\text { segurança de seus usuários e a facilidade de caminhar à noite } 52,59,60\end{array}$ & Sim \\
\hline $\begin{array}{l}\text { Paradas de transporte } \\
\text { público }\end{array}$ & $\begin{array}{c}\text { Maior densidade de paradas e estações de ônibus e metrô afeta positivamente a capacidade de } \\
\text { locomoção porque o transporte público mais elevado sugere uma infraestrutura urbana bem } \\
\text { desenvolvida } 9,52,61\end{array}$ & Não \\
\hline Presença de calçadas & $\begin{array}{l}\text { A presença de calçadas pode influenciar a prática de atividade física, principalmente a caminhada, já que } \\
\text { facilita os deslocamentos por oferecer segurança e atratividade } 54,55,62\end{array}$ & Sim \\
\hline $\begin{array}{l}\text { Qualidade das } \\
\text { calçadas }\end{array}$ & $\begin{array}{c}\text { Uma infraestrutura de calçadas de boa qualidade suporta a caminhada como uma opção de modo } \\
\text { viável e estimula a atividade física saudável. Degraus na calçada, piso escorregadio e má conservação } \\
\text { estão entre as principais causas de acidentes de pedestres } 63,64\end{array}$ & Não \\
\hline Segurança viária & $\begin{array}{c}\text { A infraestrutura necessária para aumentar a segurança de pedestres e bicicletas na presença de tráfego } \\
\text { como espaço/infraestrutura (por exemplo, calçadas, ciclovias), baixo volume de tráfego, faixas de } \\
\text { pedestres, incentivam a caminhada } 65,66\end{array}$ & Não \\
\hline Uso misto do solo & $\begin{array}{l}\text { O nível de integração dentro de uma determinada área de diferentes tipos de usos para o espaço físico, } \\
\text { incluindo residencial, escritório, varejo/comercial e espaço público. As pessoas que vivem perto de } \\
\text { múltiplas e diversificadas oportunidades de varejo tendem a fazer viagens de compras mais frequentes, } \\
\text { mais especializadas e mais curtas, muitas a pé } 58,67,68\end{array}$ & Não \\
\hline
\end{tabular}

\section{Validade preditiva}

Dos 499 idosos estudados, a maioria 53,9\% $(\mathrm{n}=269)$ era do sexo feminino. A idade média foi de 70,53 $(\mathrm{DP}=7,84)$ anos. A mediana de renda foi de R\$ 700,00. A Tabela 2 apresenta os coeficientes bruto e ajustado por sexo e idade e seus IC95\% para a associação entre índice de caminhabilidade e escore de incapacidade funcional.

Verificou-se uma associação inversa entre a caminhabilidade e o maior escore de incapacidade funcional em idosos no modelo 1, que permaneceu significante no modelo ajustado por idade, sexo e renda. Ou seja, independentemente da idade, renda e do sexo, os idosos que residiam em regiões urbanas com o maior índice de caminhabilidade apresentaram menor escore de incapacidade funcional. 


\section{Figura 1}

Distribuição espacial das variáveis candidatas ao índice de caminhabilidade. Viçosa, Minas Gerais, Brasil, 2010.

1a) Densidade residencial

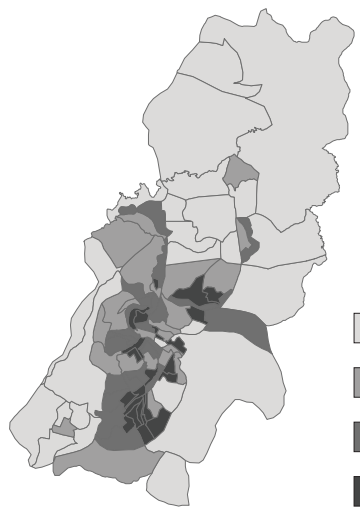

$\square, 00-0,06$

$0,07-0,10$

$0,11-0,16$

$0,17-0,30$

1d) \% de presença de calçadas
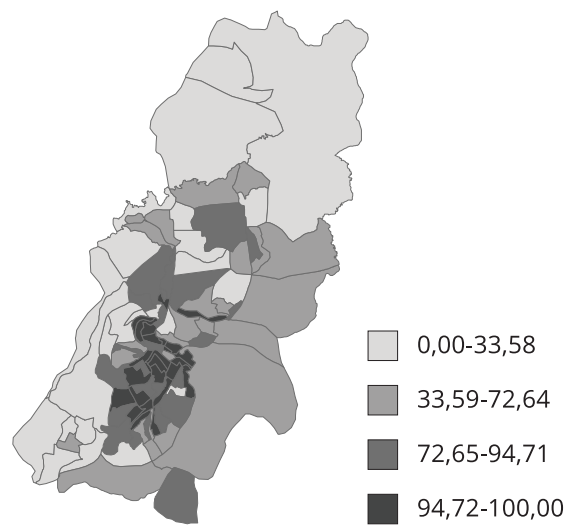

1e) Conectividade de ruas

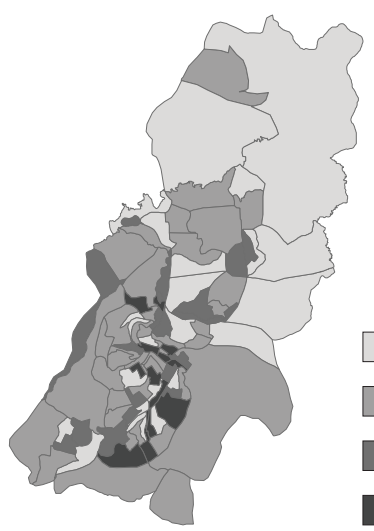

$\square$

$0,001-0,004$

$0,005-0,006$

$0,007-0,008$

$0,009-0,013$ 1c) \% de arborização

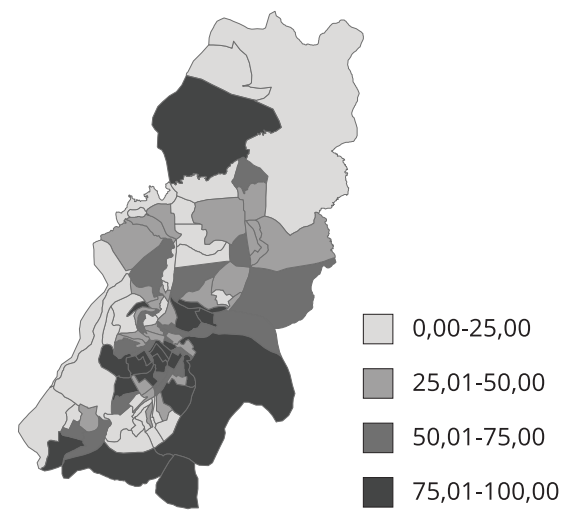

1f) \% de iluminação pública

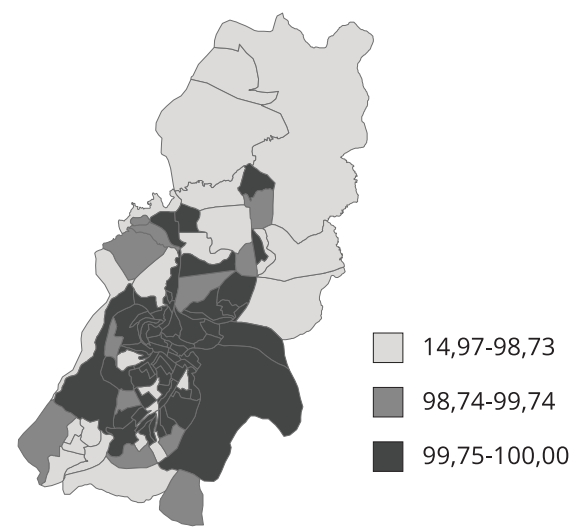

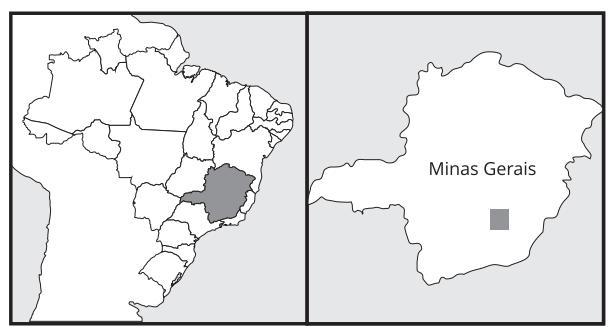


Tabela 1

Fator de carga (com rotação - varimax normalizado) para o índice de caminhabilidade.

\begin{tabular}{|c|c|c|c|}
\hline Variáveis & Fator 1 & Fator 2 & Comunalidades \\
\hline Densidade residencial & 0,763 & & 0,688 \\
\hline$\%$ de presença de calçadas & 0,752 & & 0,689 \\
\hline Densidade comercial & 0,723 & & 0,524 \\
\hline \% de presença de iluminação pública & 0,511 & & 0,364 \\
\hline Densidade de intersecções & 0,472 & & 0,223 \\
\hline \% de arborização & & 0,944 & 0,891 \\
\hline Eigenvalues & 2,15 & 1,23 & \\
\hline Explicação da variância (\%) & 35,89 & 20,43 & \\
\hline
\end{tabular}

\section{Discussão}

O presente estudo sugeriu um índice de caminhabilidade para uma cidade de médio porte e testou sua associação com a incapacidade funcional de idosos. Tal índice foi criado com base em dados que avaliaram objetivamente o ambiente por meio de SIG. Valendo-se da análise fatorial, foi selecionado um conjunto parcimonioso de variáveis utilizando dados de livre acesso para todo o país. O índice obtido permite a avaliação do ambiente das cidades como um todo e a observação particular de quais características do ambiente podem ser modificadas de forma melhorar a infraestrutura para caminhada.

Nos últimos anos, foram criados diferentes índices para avaliar a caminhabilidade de uma área. Por exemplo, o índice de walkability definido por Frank et al. 8 em 2010, o mais utilizado, consiste em quatro componentes - densidade de interseções das ruas, densidade residencial líquida, uso misto da terra e área líquida de varejo - já testados em diferentes populações e países. Mas, com exceção da densidade de interseções das ruas, são exigidos dados de registro de imóveis, incluindo limite de propriedade e uso da terra de cada parcela para todas as áreas públicas e privadas. Esses dados geográficos são de difícil obtenção não apenas em países de baixa renda, mas também em países de alta renda 33,34. Essa é uma limitação importante que impede a aplicação de tais índices objetivos no desenho urbano e na prática da saúde pública. Assim, os pesquisadores têm investido esforços para criar índices facilmente aplicáveis, como o WalkScore, que é uma ferramenta prontamente disponível que permite que projetistas urbanos, autoridades governamentais e profissionais de saúde pública identifiquem a capacidade de locomoção de áreas locais sem depender de dados geográficos detalhados ou pessoal especializado em ferramentas de dados geográficos 17. Porém, tal ferramenta é um indicador geral que não fornece informações sobre quais aspectos específicos do ambiente necessitam ser modificados para promover a caminhada. Ainda, há iniciativas de criação de índices de caminhabilidade no país 10,35 , porém estes índices utilizam dados de difícil aquisição pelos municípios, uma vez que exigem uma coleta de dados demorada e de alto custo.

Em um contexto de recessão e escassez de recursos para a pesquisa, a coleta de dados nacionais que implique investimento de tempo e dinheiro torna-se dificultada. Apenas algumas capitais brasileiras têm dados mais detalhados de suas cidades, mas a grande maioria conta apenas com os dados disponíveis do Censo Demográfico. Nesse contexto, destaca-se a importância de novas formas de análise ou ferramentas analíticas que permitam uma boa aproximação da realidade para caracterizar a caminhabilidade de um ambiente, com baixo custo e sem grande dificuldade operacional. $O$ índice aqui desenvolvido tem tais características e permite um mapeamento das cidades de forma a identificar áreas de maior vulnerabilidade no que tange à capacidade de locomoção a pé ou de bicicleta. Tal forma de transporte e atividade física está relacionada com a manutenção da capacidade funcional de idosos 36,37 .

No presente estudo, verificou-se o predomínio de áreas de baixa caminhabilidade, atribuído especialmente à baixa cobertura de iluminação e calçadas. Tais áreas estão concentradas nas regiões 


\section{Figura 2}

Espacialização do índice de caminhabilidade. Viçosa, Minas Gerais, Brasil, 2010.


Índice de caminhabilidade

Muito baixo

Baixo

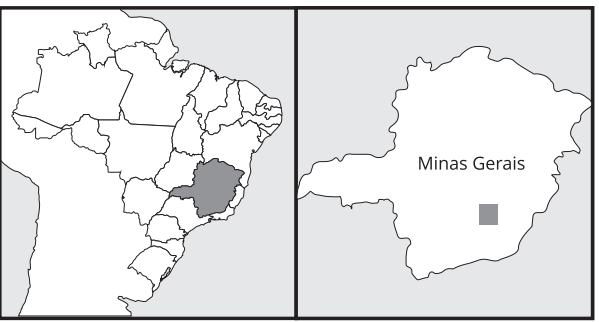

$\square$ Médio

Alto

Muito alto 
Tabela 2

Modelos de regressão para a associação entre índice de caminhabilidade e escore de imobilidade com base nas equações de estimativa generalizada (GEE).

\begin{tabular}{ccccccc}
\hline & Modelo 1 & & Modelo 2 \\
IC95\% & Valor de $\mathbf{p}$ & $\boldsymbol{\beta}$ ajustado & IC95\% & Valor de $\mathbf{p}$ \\
\hline Caminhabilidade & $-0,055$ & $-0,106 ;-0,003$ & $\mathbf{0 , 0 4}$ & $-0,024$ & $-0,046 ;-0,002$ & $\mathbf{0 , 0 3}$ \\
\hline
\end{tabular}

IC95\%: intervalo de 95\% de confiança.

Nota: Modelo 1: sem ajuste; Modelo 2: ajustado por sexo, idade e renda.

periféricas da cidade, que são as de menor renda. A exceção ocorre nas áreas dos condomínios fechados, o que reafirma a condição de privação e segregação urbana. A precariedade urbana inibe o desenvolvimento local criando um ciclo de segregação ${ }^{38}$. Dessa forma, é urgente que se priorize reformas e novas condições de uso a todos os espaços, especialmente àqueles mais necessitados, levando o direito à cidade a todos. Tais ações podem promover melhores condições de vida à população idosa, assim como para toda a população.

Ressalta-se que os espaços exteriores e equipamentos públicos são essenciais para a caminhada, independência funcional e qualidade de vida dos idosos, e afetam sua capacidade de "envelhecer no seu próprio lugar” 6,39. As barreiras físicas frequentemente desanimam os idosos a saírem de suas casas, restringindo o seu ir e vir. Dessa forma, o papel de infraestrutura adequada, tais como calçadas e locais públicos acessíveis e seguros, são alguns dos elementos da espacialidade urbana que devem ser observados para que o envelhecimento saudável e ativo seja estimulado.

Um dos maiores desafios em utilizar o SIG está na aquisição de dados detalhados que contenham informações de atributos e localização de variáveis do ambiente construído. Por exemplo, os dados sobre o uso misto do solo estão frequentemente indisponíveis para calcular a proporção da área ocupada por estabelecimentos comerciais, principalmente em municípios de menor porte 33 . Neste estudo, como medida alternativa, foi avaliada a densidade de comércios e serviços em cada setor censitário. No entanto, dados sobre segurança no trânsito, segurança relacionada ao crime, declividade e qualidade de calçadas não foram avaliados devido ao fato de não haver bancos de livre acesso e baixa dificuldade operacional disponíveis. Por esse motivo, a construção de índices e a aplicação de atributos alternativos disponíveis nacionalmente podem permitir uma ampliação do uso de índices de caminhabilidade nas pesquisas e no planejamento das cidades. A metodologia sugerida aqui tem potencial de ser aplicada em todos os municípios brasileiros.

Por outro lado, inúmeras vantagens no uso de SIG podem ser apontadas. Sua capacidade para avaliar o ambiente usando dados amplamente disponíveis tem um alto potencial por reduzir os custos, tempo de aplicação e dificuldades operacionais relacionadas à aplicação de auditorias que necessitam de trabalho de campo. Também há vantagem na utilização de dados objetivos em relação à informação subjetiva, obtida por meio de questionários aplicados aos usuários do ambiente. Os dados do SIG não apresentam viés devido a fatores que poderiam modificar a percepção dos idosos em relação ao local em que vivem, como por exemplo, os fatores emocionais 40. Adicionalmente, com a informação objetiva há uma definição clara de espaço a ser modificado e/ou estudado pelos gestores (setor censitário).

Outra análise inédita no contexto brasileiro foi a validação preditiva do índice construído. No contexto avaliado, a associação significante entre a caminhabilidade e o escore de incapacidade funcional, mesmo após ajuste por variáveis individuais como sexo, idade e renda, levanta a hipótese da importância potencial do ambiente físico da vizinhança em manter a capacidade funcional dos idosos e promover o envelhecimento saudável e ativo no nível populacional 4 . Corroborando a literatura, os resultados do presente estudo demonstram que o ambiente construído pode desempenhar um papel importante na saúde das pessoas idosas, ajudando-as a preservar sua capacidade de caminhar, independência e qualidade de vida por meio da caminhabilidade 7,12,13,14. Para a melhor avaliação do índice seria interessante estimar a sua associação com a caminhada em idosos. No entanto, devido a limitações na coleta de dados tal análise não foi possível. 
Recentemente, em abril de 2018, o Brasil lançou a Estratégia Brasil Amigo da Pessoa Idosa 41, iniciativa que tem como objetivo incentivar as comunidades e cidades a implantarem políticas públicas voltadas para a população idosa, de forma a promover o envelhecimento ativo, saudável, cidadão e sustentável. Dentro das etapas previstas para a adesão da cidade à estratégia, encontra-se o diagnóstico das condições das localidades onde as pessoas envelhecem, quando são incluídos aí o ambiente físico e a mobilidade. Esse diagnóstico visa a subsidiar a elaboração dos planos municipais de ações, nos quais constarão as ações a serem implantadas e/ou aprimoradas em períodos específicos com vistas a promover o envelhecimento ativo. Assim, o índice de caminhabilidade aqui proposto pode oferecer aos gestores das cidades e profissionais de saúde uma ferramenta simples para subsidiar a construção de vizinhanças e cidades amigáveis aos idosos.

\section{Conclusões}

O índice de caminhabilidade é uma estratégia analítica para sistematizar as principais variáveis do ambiente construído, consideradas relevantes para que uma cidade favoreça o deslocamento a pé e de bicicleta.

A construção do índice permitiu entender a relação entre os atributos físicos que potencializam o caminhar e o deslocamento cotidiano da população, evidenciando áreas prioritárias para intervenções e identificando quais características necessitam de modificações na área analisada. No contexto de uma cidade de médio porte do interior do Brasil, identificou-se como áreas prioritárias as regiões mais distantes do centro da cidade. Adicionalmente, as características presença de calçadas, densidade residencial, densidade comercial e arborização demandam modificações nessas áreas para torná-las mais atrativas.

Os resultados também indicam que o índice de caminhabilidade criado foi adequado para avaliar o potencial do ambiente físico da vizinhança em promover a maior capacidade funcional, visto que evidenciou-se associação entre pior escore de caminhabilidade e incapacidade funcional.

No entanto, as características do município podem não representar a totalidade dos municípios de médio porte e limitam a validade externa dos resultados. Em trabalhos futuros, o índice de caminhabilidade aqui proposto poderá ser usado para avaliar as relações entre caminhabilidade e a incapacidade funcional em amostras regionalmente ou nacionalmente representativas, de forma a se ampliar o seu uso em outros municípios brasileiros.

\section{Colaboradores}

A. S. F. Vegi e A. Q. Ribeiro trabalharam no desenho do estudo, análise e interpretação dos dados, redação do artigo, revisão crítica relevante do conteúdo intelectual e aprovação final da versão a ser publicada. E. I. Fernandes Filho colaborou com a análise e interpretação dos dados, redação do artigo e revisão crítica relevante do conteúdo intelectual. M. C. Pessoa e K. L. Ramos colaboraram na revisão crítica relevante do conteúdo intelectual.

\section{Informações adicionais}

ORCID: Aline Siqueira Fogal Vegi (0000-00020462-5169); Elpídio Inácio Fernandes Filho (00000003-2440-8329); Milene Cristine Pessoa (00000002-1053-5450); Karla Lisboa Ramos (0000-00021484-6328); Andréia Queiroz Ribeiro (0000-00016546-1252).

\section{Agradecimentos}

À Coordenação de Aperfeiçoamento de Pessoal de Nível Superior (Capes), Conselho Nacional de Desenvolvimento Científico e Tecnológico (CNPq) e Fundação de Amparo à Pesquisa do Estado de Minas Gerais (FAPEMIG). 


\section{Referências}

1. World Health Organization. World report on ageing and health. Geneva: World Health Organization; 2015.

2. Tuckett AG, Banchoff AW, Winter SJ, King AC. The built environment and older adults: a literature review and an applied approach to engaging older adults in built environment improvements for health. Int J Older People Nurs 2017; 13:e12171.

3. Clarke P, Nieuwenhuijsen ER. Environments for healthy ageing: a critical review. Maturitas 2009; 64:14-9.

4. Organização Mundial da Saúde. Guia global: cidade amiga do idoso. Geneva: Organização Mundial da Saúde; 2008.

5. Organización Mundial de la Salud. Acción multisectorial para un envejecimiento saludable basado en el ciclo de vida: proyecto de estrategia y plan de acción mundiales sobre el envejecimiento y la salud. 69a Asamblea Mundial de la Salud. Geneva: Organización Mundial de la Salud; 2016.

6. World Health Organization. Global strategy and action plan on ageing and health. Geneva: World Health Organization; 2017.

7. Van Holle V, Van Cauwenberg J, Van Dyck D, Deforche B, Van de Weghe N, De Bourdeaudhuij I. Relationship between neighborhood walkability and older adults' physical activity: results from the Belgian Environmental Physical Activity Study in Seniors (BEPAS Seniors). Int J Behav Nutr Phys Act 2014; 11:110.

8. Frank LD, Sallis JF, Saelens BE, Leary L, Cain $\mathrm{K}$, Conway TL, et al. The development of a walkability index: application to the Neighborhood Quality of Life Study. Br J Sports Med 2010; 44:924-33.

9. Ewing R, Cervero R. Travel and the built environment. J Am Plan Assoc 2010; 76:265-94.

10. Siqueira Reis R, Hino AAF, Ricardo Rech C, Kerr J, Curi Hallal P. Walkability and physical activity: findings from Curitiba, Brazil. Am J Prev Med 2013; 45:269-75.

11. Hajna S, Ross NA, Brazeau AS, Bélisle P, Joseph L, Dasgupta K. Associations between neighbourhood walkability and daily steps in adults: a systematic review and meta-analysis. BMC Public Health 2015; 15:768.

12. Barnett DW, Barnett A, Nathan A, Van Cauwenberg J, Cerin E; Council on Environment and Physical Activity (CEPA) - Older Adults Working Group. Built environmental correlates of older adults' total physical activity and walking: a systematic review and meta-analysis. Int J Behav Nutr Phys Act 2017; 14:103.

13. Van Cauwenberg J, Nathan A, Barnett A, Barnett DW, Cerin E, Council on Environment and Physical Activity (CEPA) - Older Adults Working Group. Relationships between neighbourhood physical environmental attributes and older adults' leisure-time physical activity: a systematic review and meta-analysis. Sport Med 2018; 48:1635-60.
14. King A, Sallis JJF, Frank LD, Saelens BBE, Cain $\mathrm{KL}$, Conway TL, et al. Aging in neighborhoods differing in walkability and income: associations with physical activity and obesity in older adults. Soc Sci Med 2011; 73:1525-33.

15. Berke EM, Koepsell TD, Moudon AV, Hoskins RE, Larson EB. Association of the built environment with physical activity and obesity in older persons. Am J Public Health 2007; 97:486-92.

16. Chor D, Cardoso LO, Nobre AA, Griep RH, Fonseca MJM, Giatti L, et al. Association between perceived neighbourhood characteristics, physical activity and diet quality: results of the Brazilian Longitudinal Study of Adult Health (ELSA-Brasil). BMC Public Health 2016; 16:751

17. Carr LJ, Dunsiger SI, Marcus BH. Walk scoreTM as a global estimate of neighborhood walkability. Am J Prev Med 2010; 39:460-3.

18. Maghelal PKP, Capp CJ. Walkability: a review of existing pedestrian indices. J Urban Reg Inf Syst Assoc 2011; 23:5-19.

19. Shashank A, Schuurman N. Unpacking walkability indices and their inherent assumptions. Health Place 2019; 55:145-54.

20. Vale DS, Saraiva M, Pereira M. Active accessibility: a review of operational measures of walking and cycling accessibility. J Transp Land Use 2016; 9:209-35.

21. Glazier RH, Weyman JT, Creatore MI, Gozdyra P, Moineddin R, Matheson FI, et al. Development and validation of an urban walkability index for Toronto, Canada. Toronto: Toronto Community Health Profiles Partnership; 2013.

22. Giles-Corti B, Mavoa S, Eagleson S, Davern $\mathrm{M}$, Roberts R, Badland $\mathrm{H}$. How walkable is Melbourne? The development of a transport walkability index for metropolitan Melbourne. Melbourne: University of Melbourne; 2015.

23. Millington C, Ward Thompson C, Rowe D, Aspinall P, Fitzsimons C, Nelson N, et al. Development of the Scottish Walkability Assessment Tool (SWAT). Health Place 2009; 15:47481.

24. Stockton JC, Duke-Williams O, Stamatakis E, Mindell JS, Brunner EJ, Shelton NJ. Development of a novel walkability index for London, United Kingdom: cross-sectional application to the Whitehall II Study. BMC Public Health 2016; 16:416.

25. Lopes AAS, Kienteka M, Fermino RC, Reis RS. Characteristics of the environmental microscale and walking and bicycling for transportation among adults in Curitiba, Paraná State, Brazil. Cad Saúde Pública 2018; 34:e00203116.

26. Tian G, Ewing R. A walk trip generation model for Portland, OR. Transp Res Part D Transp Environ 2017; 52:340-53. 
27. Instituto Brasileiro de Geografia e Estatística. Censo Demográfico 2010. Rio de Janeiro: Instituto Brasileiro de Geografia e Estatística; 2010.

28. Silva AR, Carmo MI, Alvarenga SC, Cruz TA. Retrato social de Viçosa III. Viçosa: CENSUS; 2010.

29. Instituto Brasileiro de Geografia e Estatística. Metodologia do Censo Demográfico 2010. Relatórios metodológicos. Rio de Janeiro: Instituto Brasileiro de Geografia e Estatística; 2013.

30. Laros JA. O uso da análise fatorial: algumas diretrizes para pesquisadores. In: Pasquali L, editor. Análise fatorial para pesquisadores. Brasília: LabPAM Saber e Tecnologia; 2012. p. 141-60.

31. Nascimento CDM, Ribeiro AQ, Cotta RMM, Acurcio FDA, Peixoto SV, Priore SE, et al. Factors associated with functional ability in Brazilian elderly. Arch Gerontol Geriatr 2012; 54:e89-94.

32. Lange C, Silverman EK, Xu X, Weiss ST, Laird NM. A multivariate family-based association test using generalized estimating equations: FBAT-GEE. Biostatistics 2003; 4:195-206.

33. Health Council of The Netherlands Dutch Advisory Council for Research on Spatial Planning Nature and the Environment. Nature and health: the influence of nature on social, psychological and physical well-being. The Hague: Health Council of the Netherlands/RMNO; 2004.

34. Sarkar C, Webster C, Pryor M, Tang D, Melbourne S, Zhang X, et al. Exploring associations between urban green, street design and walking: results from the Greater London boroughs. Landsc Urban Plan 2015; 143:112-25.

35. Cervero R, Duncan M. Walking, bicycling, and urban landscapes: evidence from the San Francisco Bay Area. Am J Public Health 2003; 93:1478-83

36. Sehatzadeh B, Noland RB, Weiner MD. Walking frequency, cars, dogs, and the built environment. Transp Res Part A Policy Pract 2011; 45:741-54.

37. Wells NM, Yang Y. Neighborhood design and walking. A quasi-experimental longitudinal study. Am J Prev Med 2008; 34:313-9.

38. Oakes JM, Forsyth A, Schmitz KH. The effects of neighborhood density and street connectivity on walking behavior: The Twin Cities walking study. Epidemiol Perspect Innov 2007; 4:16

39. Berrigan D, Pickle LW, Dill J. Associations between street connectivity and active transportation. Int J Health Geogr 2010; 9:20.

40. Li F, Fisher KJ, Brownson RC, Bosworth M. Multilevel modelling of built environment characteristics related to neighbourhood walking activity in older adults. J Epidemiol Community Health 2005; 59:558-64.

41. Leslie E, Coffee N, Frank LD, Owen N, Bauman AE, Hugo G. Walkability of local communities: using geographic information systems to objectively assess relevant environmental attributes. Health Place 2007; 13:111-22.
42. Loukaitou-Sideris A. Is it safe to walk? Neighborhood safety and security considerations and their effects on walking. J Plan Lit 2006; 20:219-32.

43. Van Cauwenberg J, Clarys P, De Bourdeaudhuij I, Van Holle V, Verte D, De Witte N, et al. Older adults' transportation walking: a crosssectional study on the cumulative influence of physical environmental factors. Int J Health Geogr 2013; 12:37.

44. Meeder M, Aebi T, Weidmann U. The influence of slope on walking activity and the pedestrian modal share. Transportation Research Procedia 2017; 27:141-7.

45. Rodríguez DA, Joo J. The relationship between non-motorized mode choice and the local physical environment. Transp Res Part D Transp Environ 2004; 9:151-73.

46. Lee C, Moudon AV. Correlates of walking for transportation or recreation purposes. J Phys Act Health 2006; 3(s1):S77-98.

47. Glazier RH, Creatore MI, Weyman JT, Fazli G, Matheson FI, Gozdyra P, et al. Density, destinations or both? A comparison of measures of walkability in relation to transportation behaviors, obesity and diabetes in Toronto, Canada. PLoS One 2014; 9:e85295.

48. Forsyth A, Oakes JM, Schmitz KH, Hearst M. Does residential density increase walking and other physical activity? Urban Stud 2007; 44:679-97.

49. Rodríguez DA, Evenson KR, Diez Roux AV, Brines SJ. Land use, residential density, and walking. The multi-ethnic study of atherosclerosis. Am J Prev Med 2009; 37:397-404.

50. Farrington DP, Welsh BC. Improved street lighting and crime prevention. Justice Q 2002; 19:313-42.

51. Haans A, de Kort YAW. Light distribution in dynamic street lighting: two experimental studies on its effects on perceived safety, prospect, concealment, and escape. J Environ Psychol 2012; 32:342-52.

52. Brownson RC, Hoehner CM, Day K, Forsyth A, Sallis JF. Measuring the built environment for physical activity: state of the science. Am J Prev Med 2009; 36(4 Suppl):S99-123.e12.

53. Shay E, Rodriguez DA, Cho G, Clifton KJ, Evenson KR. Comparing objective measures of environmental supports for pedestrian travel in adults. Int J Health Geogr 2009; 8:62.

54. Landis BW, Vattikuti VR, Ottenberg RM, McLeod DS, Guttenplan M. Modeling the roadside walking environment: pedestrian level of service. Transp Res Rec 2001; 1773:82-8.

55. Frackelton A, Grossman A, Palinginis E, Castrillon F, Elango V. Measuring walkability: development of an automated sidewalk quality assessment tool. Suburban Sustainability 2013; $1: 4$.

56. Dumbaugh E. Designing communities to enhance the safety and mobility of older adults. J Plan Lit 2008; 23:17-36. 
57. McGinn AP, Evenson KR, Herring AH, Huston SL, Rodriguez DA. Exploring associations between physical activity and perceived and objective measures of the built environment. J Urban Health 2007; 84:162-84.

58. Christian H, Bull FCL, Middleton NJ, Knuiman M, Divitini ML, Hooper P, et al. How important is the land use mix measure in understanding walking behaviour? Results from the Reside study. Int J Behav Nutr Phys Act 2011; 8:55.

59. Chum A, Atkinson P, O'Campo P. Does time spent in the residential neighbourhood moderate the relationship between neighbourhood walkability and transport-related walking? A cross-sectional study from Toronto, Canada. BMJ Open 2019; 9:e023598.

60. Kerr J, Sallis JF, Owen N, De Bourdeaudhuij I, Cerin E, Sugiyama T, et al. Advancing science and policy through a coordinated international study of physical activity and built environments: IPEN adult methods. J Phys Act Health 2013; 10:581-601.

61. Salvo D, Reis RS, Sarmiento OL, Pratt M. Overcoming the challenges of conducting physical activity and built environment research in Latin America: IPEN Latin America. Prev Med (Baltim) 2014; 69:S86-92.
62. Motomura MCN, Fontoura LC, Kanashiro M Understanding walkable areas: applicability and analysis of a walkability index in a Brazilian city. Ambiente Construído 2018; 18:41325.

63. Rosso AL, Auchincloss AH, Michael YLY. The urban built environment and mobility in older adults: a comprehensive review. J Aging Res 2011; 2011:816106.

64. Verbrugge LML, Jette AMA. The disablement process. Soc Sci Med 1994; 38:1-14.

65. Santos AP, Rocha SF, Abreu MVS, Calijuri ML, Santos PM. O uso da análise multicritério no mapeamento da fragilidade social da área urbanizada do Município de Viçosa-MG. Revista Brasileira de Cartografia 2012; 64:635-43.

66. Kestens Y, Chaix B, Gerber P, Desprès M, Gauvin L, Klein O, et al. Understanding the role of contrasting urban contexts in healthy aging: an international cohort study using wearable sensor devices (the CURHA study protocol). BMC Geriatr 2016; 16:96.

67. Zadra JR, Clore GL. Emotion and perception: the role of affective information. Wiley Interdiscip Rev Cogn Sci 2011; 2:676-85.

68. Ministério da Cidadania. Estratégia Brasil Amigo da Pessoa Idosa: introdução à estratégia. Brasília: Ministério da Cidadania; 2019. 


\section{Abstract}

Evidence has shown that urban environments that discourage walking contribute to functional incapacity in the elderly. Various indices have been proposed to describe an area's walkability, combining different aspects of the built environment that promote (or inhibit) walking. However, due to problems with the quality and availability of data in Brazil, there is no walkability index to date applies to all cities of the country and that has been properly tested in the population. The current study aimed to propose a walkability index based on geographic information systems for a mediumsized city, with open-access data, and to test its association with functional incapacity in the elderly. The study used data from the urban area of a medium-sized Brazilian city to select a parsimonious set of variables through factor analysis. The resulting index was tested for its association with the capacity to perform activities of daily living that require more movement, in 499 elderly, using generalized estimating equations. The resulting walkability index consists of residential density, commercial density, street connectivity, presence of sidewalks, and public lighting. These variables comprised the first factor in the factor analysis, excluding only arborization which was retained in the second factor. The worst walkability score was associated with the highest functional incapacity score. Based on the results and their validation, the study suggests an easily applicable walkability index with great potential for use in action plans to adapt environments.

Aged; Healthy Aging; Mobility Limitation; Environment and Public Health; Health of the Elderly

\section{Resumen}

Existen evidencias de que los ambientes urbanos que desestimulan los paseos contribuyen a la incapacidad funcional de los ancianos. Se propusieron varios índices para describir la posibilidad de pasear en un área, combinando aspectos del ambiente construido que promueven o inhiben los paseos. No obstante, debido a problemas de calidad y disponibilidad de datos en Brasil, hasta el momento no existe un indice sobre la posibilidad de pasear, aplicable a todas las ciudades del país, y debidamente probado en la población. El objetivo de este estudio fue proponer un indice sobre la posibilidad de pasear, basado en sistemas de información geográfica para una ciudad de tamaño medio, con datos de libre acceso, así como probar su asociación con la incapacidad funcional en ancianos. Se utilizaron los datos del área urbana de un municipio de tamaño medio para seleccionar un conjunto parsimonioso de variables mediante análisis factorial. El indice obtenido fue probado en 499 ancianos, en lo que se refiere a su asociación con la capacidad para la realización de actividades de vida diaria, que requieren un mayor movimiento, utilizando ecuaciones de estimación generalizadas. El índice resultante sobre la posibilidad de pasear estaba compuesto por: densidad residencial, densidad comercial, conectividad de calles, presencia de aceras e iluminación pública. Estas variables formaron parte del primer factor de análisis factorial, excluyendo solamente la arborización, que quedó fijada en el segundo factor. Se verificó que la peor puntuación sobre la posibilidad de pasear se asoció a la mayor puntuación de incapacidad funcional. En base a los resultados, y a la validación de los mismos, el estudio sugiere un indice sobre la posibilidad de realizar paseos, fácilmente aplicable, con un gran potencial de uso en planes de acción para adecuar los ambientes.

Anciano; Envejecimiento Saludable; Limitación de la Movilidad; Medio Ambiente y Salud Pública; Salud del Anciano
Recebido em 10/Nov/2018

Versão final reapresentada em 22/Ago/2019

Aprovado em 03/Set/2019 